\title{
Global trends of epidemiological research in livestock tuberculosis for the last four decades
}

2 Ana C. Reis ${ }^{1,2}$, Beatriz Ramos ${ }^{1,2}$, André C. Pereira ${ }^{1,2}$, Mónica V. Cunha ${ }^{1,2, *}$

$4{ }^{1}$ Centre for Ecology, Evolution and Environmental Changes (cE3c), Faculdade de Ciências, 5 Universidade de Lisboa, 1749-016 Lisboa, Portugal.

$6 \quad{ }^{2}$ Biosystems \& Integrative Sciences Institute (BioISI), Faculdade de Ciências, Universidade 7 de Lisboa, 1749-016 Lisboa, Portugal.

8 * Correspondence:

9 Mónica V. Cunha, Faculdade de Ciências, Universidade de Lisboa, Campo Grande, 1749$10 \quad 016$ Lisboa. mscunha@fc.ul.pt

11

12 Keywords: animal tuberculosis, livestock, epidemiology, one health, bibliometric review, 13 research trends, livestock production system

14

15 Running title: Epidemiological research in livestock TB

16

17 Reis, AC, Ramos, B, Pereira, AC, Cunha, MV. Global trends of epidemiological research

18 in livestock tuberculosis for the last four decades. Transbound Emerg Dis. 2020; 00: 1- 14.

19 https://doi.org/10.1111/tbed.13763 


\section{Abstract}

22 Animal tuberculosis (TB) caused by Mycobacterium tuberculosis complex (MTC) bacteria 23 remains as one of the most significant infectious diseases of livestock, despite decades of 24 eradication programs and research efforts, in an era where the livestock sector is amongst the 25 most important and rapidly expanding commercial agricultural segments worldwide.

26 This work provides a global overview of the spatial and temporal trends of reported scientific 27 knowledge of TB in livestock, aiming to gain insights into research subtopics within the 28 animal TB epidemiology domain and to highlight territorial inequalities regarding data 29 reporting and research outputs over the years. To deliver such information, peer-reviewed 30 reports of TB studies in livestock were retrieved from the Web of Science and Google 31 Scholar, systematized, and dissected. The validated dataset contained 443 occurrence 32 observations, covering the 1981-2020 period (39 years).

33 We highlight a clear move towards transdisciplinary areas and the One Health approach, with 34 a global temporal increase of publications combining livestock with wildlife and/or human 35 components, which reflect the importance of non-prototypical hosts as key to understanding 36 animal TB. It becomes evident that cattle is the main host across works from all continents, 37 however many regions remain poorly surveyed. TB research in livestock in low-/middle38 income countries is markedly growing, reflecting changes in animal husbandry, but also 39 mirroring the globalization era, with a marked increase in international collaboration and 40 capacitation programs for scientific and technological development.

41 This review gives an overview of the most prolific continents, countries, and research fields 42 in animal TB epidemiology, clearly outlining knowledge gaps and key priority topics. The 43 estimated growth trend of livestock production until 2050, particularly in Asia and Africa, in 44 response to human population growth and animal-protein demand, will require further 45 investment in early surveillance and adaptive research to accommodate the higher diversity 46 of livestock species and MTC members and raising the possibility to finetune funding 47 schemes. 


\section{Introduction}

50 Tuberculosis (TB) is caused by a group of pathogenic mycobacteria grouped in the Mycobacterium tuberculosis complex (MTC). This is a chronic, slow-progression disease that can lead to granuloma formation, mainly in the lymph nodes and lungs (Palmer \& Waters, 2006).

MTC members have been described as a series of host-adapted ecotypes, with each species or ecotype exhibiting distinct host tropism, phenotype, and pathogenicity, and marked by molecular singularities (Brites et al., 2018; Gagneux, 2018; Smith et al., 2006). Mycobacterium tuberculosis, Mycobacterium africanum, and Mycobacterium canettii are almost exclusively human pathogens, while the other ecotypes are animal-adapted complex members. Mycobacterium bovis is considered to have the widest host range: ungulates, carnivores, marsupials, rodents, lagomorphs, and primates, humans included (RodriguezCampos, Smith, Boniotti, \& Aranaz, 2014; Thoen, Lobue, \& de Kantor, 2006). Mycobacterium caprae was first described as main the causative agent of TB in goats (Aranaz, Cousins, Mateos, \& Dominguez, 2003), however, the pathogen was already isolated from other mammal species, including wildlife and humans, mainly in European countries (Reis, Albuquerque, Botelho, \& Cunha, 2020; Rodríguez et al., 2011). As for the remaining members, Mycobacterium microti natural hosts are rodents (Kipar et al., 2014); Mycobacterium pinnipidii is associated with infection in pinnipeds, such as sea lions (Cousins et al., 2003); Mycobacterium mungi was described in banded mongooses from Southern Africa (Alexander et al., 2010); and Mycobacterium orygis was originally isolated from African and Asian ungulates (van Ingen et al., 2012). Two recent additions to the MTC are still not formalized, the "chimpanzee bacillus" isolated from chimpanzee (Coscolla et al., 2013) and the "dassie bacillus" from hyrax (Mostowy, Cousins, \& Behr, 2004), both in Africa.

When considering livestock animal populations, M. bovis and M. caprae are, thus far, the most relevant TB-causing agents. Indeed, several countries have implemented TB eradication programs in livestock, mainly in cattle populations, with some minor exceptions also including the test and cull of small ruminants. TB control programs are currently in progress in several European countries. Portugal, Spain, the United Kingdom, Ireland, Malta, and Italy hold such programs with co-funding by the European Union (EU, 2020). Some federal states of the United States of America (USA), New Zealand, and developing countries, that still have not achieved the TB-free status, remain incessantly working for that purpose also under test and slaughter schemes.

The livestock sector continues to evolve on a global scale, being one of the agricultural subsectors that presents a more pronounced growing rate in developing countries (Thornton, 2010). Global livestock production has increased substantially since the 1960s and is expected to grow steadily for the next decades as a consequence of the predicted increase in the demand for animal products (Thornton, 2010). It is estimated that the human population will grow, approximately, to 9 billion in 2050 and the majority will live in urban settings, two factors that, together with the shift of diets and food consumption patterns towards animal-derived protein, will contribute to the higher demand of meat supply. The rapid development of this sector can potentiate difficulties related with the control of established or emerging diseases, especially in countries with lower economic capacity to implement 


\section{$\begin{array}{lll}131 & 2 & \text { Methods }\end{array}$}

132 This systematic review followed the recommendations provided by the PRISMA guidelines 133 (Fig. 1).

\section{$134 \quad 2.1$ Data assembly} impact on several domains. First, on animal health but, being a zoonosis, also on human health. The consumption of unpasteurized dairy products and direct close contact with infected animals in the context of occupational exposure have been attributed as potential sources of infection to humans (Cvetnic et al., 2007; Kubica, Rüsch-Gerdes, \& Niemann, 2003; Müller et al., 2013; Rodríguez et al., 2009). Second, it causes severe economic constraints, due to the costs associated with the implementation of surveillance or eradication programs; compensations to farmers due compulsory slaughter of reactors, the reduction in protein conversion yields and productivity; trade restrictions; and reduced value of live animals and animal-derived products.

TB in livestock is best characterized in the cattle population, however, some geographical disparities in data reporting and method standardization are evident. Moreover, despite there being national reference laboratories for diagnosis purposes in developed and developing countries which follow international guidelines from OIE, the number of research groups addressing this research topic with different resources, technical skills, and technological advancements increase the heterogeneity and quality of scientific production. A lack of comprehensive information concerning overall geographic disease prevalence, diagnostic techniques under routine application, genotyping methods, and transmission inference strategies subsist. Plus, the distribution of disease in livestock species regarded as nonprototypical for TB, such as goats, sheep, or pigs, or animals regarded as wildlife but which are highly managed and maintained in confined environments for economical purposes, such as touristic activities (e.g. dromedaries), transportation of humans and goods (e.g. llamas), game farms, zoological parks, and, others, remain poorly known on a global level. It could thus be useful to highlight the status and geographical distribution of epidemiological knowledge related to TB in livestock. Such global mapping is elemental to quickly identify trends, understand the relative contribution of livestock managing systems, spot the regions in which research dedicated to animal TB is intense or under expansion, and mark those territories that urgently need to generate new data. This systematization of knowledge may inform strategic decisions on when, where, and how should resources be allocated. temporal distribution of scientific works addressing the epidemiological scenario of TB in ; MTC as TB agents in livestock and their relative proportion across ecotypes; (iv) evaluate the different diagnostic and differentiation methodologies that have been implemented; (v) assess the relative contribution of livestock-wildlife and livestock-human interactions in the published articles; and (vi) identify the drivers that are meaningful to TB transmission in livestock. 
The review question was defined as "What is the current global distribution of published scientific literature of animal TB epidemiology in livestock?". To gather the peer-reviewed database, literature searches were undertaken using a highly relevant database and search engine and the resulting articles were manually reviewed. Reports by national or international authorities concerning official data submitted by each country were not considered.

\subsection{Data collection}

The literature search was conducted through ISI's Web of Science online interface (http://www.isiknowledge.com), in all available databases, and Google Scholar (Fig. 1). Search results were delimited based on the following Boolean query executed within a single search. No time and geographical location restrictions were placed on these searches, and only those published in English and Portuguese retrieved. The searches were last updated on $15^{\text {th }}$ February 2020.

The search strategy consisted of compiling three search strings, one for each category (tuberculosis, epidemiology, and livestock), and combining these by the Boolean operator "AND" to obtain only the intersection. Specifically, we used the following Boolean search statement: \#1 "tuberculosis"; \#2 "epidemiology": "epidemiology" OR "transmission"; \#3 "livestock": "Livestock" OR "Farmed" OR "Cattle" OR "Sheep" OR "Ovine" OR "Goat" OR "Pig" OR "Equine" OR "Camel" OR "Dromedary" OR "Alpaca" OR "Llama"; and the intersection consisted in \#1 AND \#2 AND \#3. The term "Farmed" was included in this search strategy to comprise all species maintained in a confined environment with human intervention, including, for example, situations of farmed game species, zoos, or animals used for touristic activities. The terms "Camel", "Dromedary", "Alpaca" and "Llama" were also included to consider specific production applications and touristic activities.

\subsection{Inclusion and exclusion criteria}

Results for all articles were imported into a bibliographic referencing tool and all query results were verified manually to exclude duplicated entries (Fig. 1). Besides, publications clearly indexed either as review, editorial, conference posters, theses, or errata were excluded (Fig. 1). Papers in languages other than English and Portuguese or inaccessible were also omitted (Fig. 1).

A preliminary screening was made based on the title and abstract content of the manuscripts resulting in the elimination of the ones that did not contain information relating to TB epidemiology in livestock, therefore articles considering experimental infection, vaccination trials or comparative and functional genomics were not included. Those articles thought to have a reasonable reflection on the review question were fully scanned and the ones not focusing on the review theme were excluded (Fig. 1 and Supplementary Table 1).

\subsection{Data analysis}

All publications were included with the following variables retrieved: publication date, publication journal, journal subject category, document type, author, organization of origin, language, author's country of origin, title, abstract, keywords, Keywords Plus, and references. A bibliometric analysis was performed on included articles using the Bibliometrix package in R (Aria \& Cuccurullo, 2017), and the bibliometric indicators were evaluated by 
authors, countries, sources, and thematic fields. The analyses of thematic fields were performed based on "Keywords Plus", indexing author' keywords for the article being indexed, but also indexing the terms derived from the titles of articles for those that did not have author' keywords. Additionally, all articles were fully analyzed and information regarding the period of study, country of study, mammalian host, infectious agent, TB transmission drivers pointed out by the authors, diagnostic and differentiation (genotyping) methods, and the top five spoligotyping profiles.

\section{Results and Discussion}

The present study outlines the bibliometric indicators and scientific knowledge of reported scientific research related to animal TB in livestock. The initial 1489 records were transferred to Endnote: the duplicates $(n=46)$, not relevant publications $(n=884)$ [e.g. publications related to Mycobacterium avium subsp. paratuberculosis or wildlife], and inaccessible publications $(n=25)$ were removed (Fig. 1). Additionally, incorrectly indexed reviews $(n=63)$, conference posters $(n=7)$, and theses $(n=19)$, together with other language publications $(n=1)$, and evidence of duplicate data $(n=1)$, were excluded. After the initial screening, a total of 443 publications were considered (Supplementary Table 1).

\subsection{Temporal evolution}

This review results in the analyses of 443 documents, which originated from 115 different sources, with a total of 2049 authors, published between 1981 and 2020 (39 years) with a medium rise of 10 articles per year. The annual scientific production has been increasing over the years since 1993, with marked growth since 2003 when the annual medium rise stands at 24 articles per year, reaching a peak between 2011 and 2019 (Fig. 2a). The study period (that is the period of time to which animal data was related) of the analyzed documents was between 1944 and 2018, with a particular increase starting in 1990, reaching a peak between 2004 and 2010 (Fig. 2b). Most studies reported a duration period of one to 10 years (74.3\%), with Oceania being the exception, wherein the majority of studies had a duration period of 11 to 20 years $(n=6)$, while long-period studies ( $>20$ years) were mainly performed in Europe $(n=15)$. The average number of citations per document was 22.32, the average number of documents per author was 0.22 , and the average of authors per document was 4.63.

These increases in studies and publications starting in 2003 could be related with the European Union (EU) funding assistance to official governmental programs to control and eradicate bovine $\mathrm{TB}$ in addition to the increasing interest of TB as a One Health paradigm disease (FAO, 2011; Gibbs, 2014). The Global Fund to Fight AIDS, TB, and Malaria created in 2002 (www.theglobalfund.org) raised awareness for the zoonotic potential of TB (FAO, 2011; Gibbs, 2014). The research agendas under the Sixth and Seventh Framework Programs (2002-2013) of the EU and under the Horizon 2020 also contributed with funding to projects under the animal TB or One Health umbrella. Operational programs by Member States of the EU taking advantage from the European Structural and Investment Funds also applied resources to the animal TB problematic during the programming period, in addition to European Territorial Cooperation, cross-border or interregional operational programs. Other national programs in the UK or USA, just to mention a few countries, also gave support to research in animal TB epidemiology. The increase of TB research in livestock can also be related with changes in animal husbandry systems and the globalization era, with a marked 
increase in international capacitation programs for scientific and technological development for under-developed countries. Other specific events could also have triggered more publications, for example, the extensive badger culling program of United Kingdom implemented between 1998 and 2005 (Bourne, 2007), which, in that period and the following years, led to an increase of the number of publications at the livestock-wildlife interface; and between 2005 and 2012, the state of Minnesota in the USA implemented a series of harsh measures in white-tailed deer and cattle to re-establish the TB-free status (Cross, Heeren, Cornicelli, \& Fulton, 2018), so several publications on this issue also came to light.

\subsection{Geographical location}

Regarding the geographic location of each publication study area, most studies were performed in Europe (number of articles $=283$ ), followed by Africa $(n=157)$, South America $(n=59)$, North America $(n=55)$, Asia $(n=29)$ and, finally, Oceania $(n=21)$. Moreover, a minority of studies report study areas in more than two countries or located in different continents $(n=10)$ or do not identify the study area $(n=7)$. During the reviewed period, the average publication in Europe was eight articles per year, followed by four articles per year in Africa, and one article per year in the remaining continents.

Taking into consideration the institutional affiliations of all authors, authors from European and American institutions hold higher productivity, with the UK (number of publications=216), Spain $(n=207)$, USA $(n=163)$, Ireland $(n=118)$ and Brazil $(n=103)$ positioning on the top five of countries with more publications. Such score was obtained by counting the country of each author's institutional affiliations within each publication. Moreover, the UK (number of citations $=2205)$, Spain $(n=1081)$, Ireland $(n=818)$, USA $(n=624)$, and Australia $(n=447)$ were the top five most cited countries. The lower citation of Brazilian productions may have been the result of country-specific research, with less transposition to other countries, together with some linguistic bias and limitations, since some articles were published in Portuguese and/or in national journals. In contrast, Australia showed lower production of original research papers but higher citation numbers, which could be attributed to the international interest in control and eradication measures being used in Oceania.

Regarding study location area, the UK (number of articles=68), Spain $(n=53)$, Ethiopia $(n=37)$, USA ( $n=29)$, Ireland $(n=24)$, Brazil $(n=24)$, and Mexico $(n=22)$, were the countries that registered more than 20 articles. These countries have reported a high burden of animal TB (EFSA \& ECDC, 2019; Ortiz et al., 2014; Sibhat et al., 2017), except for the USA and Brazil. Moreover, these countries stood out with high productivity in interface areas, incorporating the problematic of TB in wildlife populations and burden associated with the zoonotic potential of the etiological agent. The USA reports were limited to TB outbreaks in Minnesota and Michigan, mainly due to high contact frequencies between cattle and whitetailed deer (wildlife reservoir), but have considerable available resources to study the disease (Cross et al., 2018; Miller \& Sweeney, 2013; Schmitt, O'brien, Bruning-Fann, \& Fitzgerald, 2002). Whereas Brazil reports sporadic outbreaks on a nationwide scale, but with lower overall prevalence (Neto et al., 2016).

Regarding partnerships across countries, the strong collaboration networks across European, American, and African countries are evident, with a marked number of collaborations of African and Asian emerging countries with American and European ones (Fig. 3). Alliances 
between the UK and Ireland (number of collaborations=17), UK and USA ( $n=14)$, Spain and UK $(n=12)$, Ethiopia and UK $(n=11)$ and Spain and USA $(n=11)$, are the most frequent, corresponding to the origins of more published works and cited authors and countries, highlighting the importance of international collaborations to the success of scientific research outputs (Fig. 3). Thus, 30\% of the studies in Ethiopia resulted from international collaborations with the UK, which could justify the abundance of TB studies in this African country compared to its overall scientific output. Interestingly, the works from the top 3 productive countries (UK, Spain, and USA) represent approximately 50\% of international collaborations (between $45.2 \%$ (Spain) and 48.6\% (USA)). This high rate of publications could also be related to the higher availability of resources to dedicate to the animal TB problematics compared to other countries. On the other hand, the co-occurrence network analysis of authors illustrates an intense collaboration network at the national level within Spanish authors, that represent $60 \%$ of the top 10 of the most prolific authors.

Evaluating the affiliation of each author, the University Complutense of Madrid ( $n=55)$ in Spain, University College Dublin $(n=50)$ in Ireland, University of Addis Ababa $(n=30)$ in Ethiopia, University of Edinburgh $(n=27)$ and Animal and Plant Health Agency (APHA) $(n=27)$ both in the UK, and the University of Pretoria $(n=25)$ in South Africa are the most highlighted institutions publishing on animal TB in livestock. The University Complutense of Madrid houses the European Union Reference Laboratory (EURL) of bovine TB in the VISAVET health surveillance center, and the APHA is an agency of the Department for Environment, Food and Rural Affairs (DEFRA) of the UK, while the other institutions are not related to official reference laboratories or agencies.

\subsection{Sources}

Focusing on sources, the top five publishing journals in the field of livestock TB are Preventive Veterinary Medicine (number of publications=50), followed by the Journal of Clinical Microbiology (JCM) ( $n=35)$, Veterinary Microbiology $(n=26)$, PLOS ONE $(n=24)$, and, finally, Tropical Animal Health and Production (n=23) (Supplementary Fig. 1). Analyzing the dynamics of these sources over the years, a predominance of the JCM is evident until 2005, when Preventive Veterinary Medicine emerged as the major publisher (Supplementary Fig. 1). This swap could be justified by the current focus of JCM on human health-related topics. Additionally, an increase over the last few years of animal TB epidemiology publications in more broad journals have been registered. However, the top five most cited sources remain the JCM (number of citations=1625), Veterinary Microbiology $(n=1010)$, Preventive Veterinary Medicine $(n=805)$, Veterinary Record ( $n=472)$, and PLOS ONE $(n=348)$. So, despite not being one of the top publishers, the works published by Veterinary Record on livestock TB can be perceived as of high interest in this scientific field.

To study the temporal evolution of fields of publication among the 443 publications, the top five fields were scrutinized. "Veterinary sciences" is the main publication field throughout the years, following the increase in the general publication area after 2005 (Fig. 4). "Infectious diseases" is the main emergent publication field since 2011, contrary to "Microbiology" that has been decreasing in the last few years (Fig. 4). "Science and Technology - Other Topics" is also emergent due to an increase of reports in more generalized journals, such as PLOS ONE (Fig. 4). Thus, an increasing tendency of authors 
to publish in journals more related to infectious diseases, instead of general microbiology, can be perceived and, at the same time, an aptitude to publish in more global, open access, publications, in parallel with increased interest of the general scientific community in animal

310 TB, whereas earlier publications were more restricted to researchers with academic degrees

311 in veterinary medicine.

312 The top five more globally cited papers retrieved (i.e., the number of times an article that integrates the collection has been cited by any other article) are the Nature letter by Gilbert et al. (2005) (number of citations=234), one of the first reports describing large temporal and spatial data on cattle movements and their relationship with bovine TB epidemiology in Great Britain (Gilbert et al., 2005); the article reporting the use of spoligotyping as a tool for $M$. bovis differentiation by Aranaz et al. (1996) $(n=189)$ that is maintained nowadays as the goldstandard method for MTC strains genotyping and molecular epidemiology studies (Aranaz et al., 1996); the description of M. pinnipedii as a new member of MTC by Cousins et al. (2003) (n=170) (Cousins et al., 2003); the proposal of the IS6110-RFLP method by Van Soolingen et al. (1994) $(n=131)$ that was used for several years as the gold-standard for genotyping of MTC isolates, including M. bovis (van Soolingen, de Haas, Hermans, \& van Embden, 1994); and the article by Smith et al. (2003) (n=126) reporting the clonal expansion of $M$. bovis in Great Britain, essential for the assembly of predictive evolutionary scenarios that have been produced ever since (Smith et al., 2003).

The top five more locally cited references (i.e., the number of times a document from the collection under analysis has been cited by other documents in this same collection) includes: firstly, the article reporting the use of spoligotyping as a tool for $M$. bovis differentiation by Aranaz et al. (1996) (number of citations=58) (Aranaz et al., 1996); the article by Haddad $e t$ al. (2001), regarding spoligotype diversity in French isolates ( $n=50)$ (Haddad et al., 2001); the article by Gilbert et al. (2005), describing the interactions between cattle movements and bovine TB in Great Britain ( $n=36$ ) (Gilbert et al., 2005); an article evaluating MIRU-VNTR epidemiological relevance and establishing the comparison with RFLP by Allix et al. (2006) ( $n=33)$ (Allix et al., 2006); and finally, the article describing the spoligotype diversity of $M$. bovis and M. caprae strains isolated from different animals in Portugal by Duarte et al. (2008) $(n=31)$ (Duarte, Domingos, Amado, \& Botelho, 2008).

This panel of papers reflects the generalized interest of the scientific community in obtaining a deeper knowledge of MTC members' population structure and diversity and to go on building hypothetical transmission networks.

Two clusters result from the co-citation network analysis built on global citation data (i.e. the full set of references cited by the analyzed papers), which put (Kamerbeek et al., 1997) and (Cosivi et al., 1998) as the central articles of each of these clusters (Supplementary Fig. 2). The first cluster comprises several articles approaching different aspects of mycobacterial molecular evolution, including MIRU-VNTR method description (Frothingham \& MeekerO'Connell, 1998), the evolutionary scenario of MTC (Brosch et al., 2002), as well as the phylogeography of the M. bovis clonal complexes (Smith, 2012), among others (Supplementary fig. 2). The latter cluster is mainly composed of review articles about the different aspects of epidemiology and transmission of MTC, particularly M. bovis (e.g. (Ayele et al., 2004; Corner, 2006; Humblet, Boschiroli, \& Saegerman, 2009)) (Supplementary Fig. 2). 


\subsection{Thematic}

352

353

354

355

356

357

358

359

360

361

362

363

364

365

366

367

368

369

370

371

372

373

374

375

376

377

378

379

380

381

382

383

384

385

386

387

388

389

390

391

392

393

The top 10 most frequently found terms in Keywords Plus were "epidemiology" (number of occurrences=163), "Mycobacterium bovis" $(n=130)$, "cattle" $(n=122)$, "infection" $(n=121)$, "transmission" ( $n=87)$, "diagnostic" $(n=80)$, "tuberculosis" $(n=77)$, "differentiation" $(n=62)$, "animals" $(n=58)$, and "identification" $(n=55)$ (Supplementary Fig. 3). The words match the focus of the research on the transmission and epidemiology of animal TB in livestock, however, it is perceivable that most works are based on the diagnostic of animal TB, followed by the differentiation (genotyping, to be more accurate) and, finally, the identification of MTC strains. Additionally, the co-occurrence network analysis of Keyword Plus resulted in the establishment of two clusters, one composed by words related to molecular epidemiology, such as identification and differentiation (i.e. genotyping) of MTC strains, while the other cluster is composed of words related to disease transmission risk factors and diagnosis.

According to the description of samples from wildlife and/or human origin in association with samples from livestock, the articles were divided into four groups: livestock, livestockwildlife, livestock-human, or livestock-wildlife-human. The majority were exclusively livestock-related (64.1\%), followed by the livestock-wildlife components (23.5\%), livestockhuman interface $(8.6 \%)$, and, finally, highlighting the One Health perspective at the interfaces of livestock, wildlife, and humans (3.8\%). In this assessment, both the livestockwildlife interface and the zoonosis-related studies have been increasing since 2004, coupled with the increasing number of cattle-related studies, contributing to global expansion in animal TB-related studies (Fig. 5a). A clear accumulation in the publication rate of studies focusing on the interface between livestock and wildlife, of 3 articles per year, was registered.

Livestock-specific studies are the most reported across all continents (ranging from $42 \%$ in North America to $7 \%$ in Asia), except the Oceania continent where livestock is usually studied together with the wildlife (45.0\%) or with wildlife and human components (45.0\%) (Fig. 5b). Studies focusing on the livestock-wildlife interface were also mainly conducted in Europe (40.6\%) (Fig. 5b). In these two continents, since the end of the $20^{\text {th }}$ century, the acknowledgment of wildlife TB reservoirs has been consolidated (Cooke, Jackson, Coleman, \& Alley, 1995; Gallagher, Monies, Gavier-Widen, \& Rule, 1998) and animal TB became most frequently tackled from the multi-host, wildlife-livestock, perspective, progressively driving more interface studies, while the addition of the One Health perspective led to the inclusion of humans into the equation (FAO, 2011; Gibbs, 2014). However, the majority of studies focusing on the zoonotic potential of animal TB remain in Africa (18.3\%) and North America (15.3\%) (Fig. 5b). Africa seems to be the continent were the zoonotic and reverse zoonotic (i.e. zooanthroponotic) dimensions of TB appear more frequently as a concern, together with North America. While socioeconomic and cultural factors are evident for Africa prominence on this subject, given the great physical proximity and interaction of humans with animals, the reasons behind North America centering on the zoonotic component are related to the origin of many studies from Mexico. In African countries, and to a lesser extent, in Mexico, livestock production is mainly based on small farms, where often the animals are kept near their owners, with the additional problem of raw milk and raw milk-derived product consumption (Carruth et al., 2016). These factors increase the risk of exposure to TB both in the cases of zoonosis and reverse zoonosis.

\subsection{Diagnostic and genotyping methodologies}


The analysis of the most frequent diagnostic and genotyping methods within the publication dataset scrutinized in this review was conducted. In the first group, histopathology (32.4\%), tuberculin-based diagnosis (29.8\%) and nucleic acid-based methods (24.6\%) were the most prevalent (Fig. 6a). Furthermore, about half of works included a bacteriological isolation step in their workflow, and when consider nucleic acid and biochemical identification methods, culture was performed as prior step in over $90 \%$ of works. Bacteriological culture and histopathology diagnosis remain the reference methodologies to confirm a diagnosis for animal TB in vitro (OIE, 2018), this high occurrence was expected. Tuberculin-based diagnosis is the most used method for in vivo diagnosis, due to its low cost-benefit, and the recognition by the OIE and the European Commission of this method as a primary screening test for the ante mortem diagnosis of TB in cattle, which also contributes for its higher applicability (OIE, 2018). The ratio between the three major diagnostic methods is similar across continents, with three exceptions: Oceania reported more tuberculin-based studies $(41.7 \%)$ and less nucleic acid-based (16.7\%); South America performed more nucleic acidbased studies (34.6\%) and less tuberculin-based ones (17.3\%); and Asia performed less histopathology-based works (22.4\%) (Fig. 6a). Interferon-gamma test was developed in 1990 , however, it is still a rarely used method for a diagnosis of animal TB $(4.3 \%)$, with no studies reported in Asia, due to its high cost, complexity, and need to process blood samples for the laboratory sensitization test within a few hours, being mainly used as an ancillary assay to clarify uncertain tuberculin test results and to give laboratory assistance to chronically infected herds. But it is not planned in control and eradication programs as a firstline diagnosis method (OIE, 2018) (Fig. 6a). Biochemical assays to identify MTC members are not commonly reported (8.9\%), once they have been replaced over the years by nucleic acid-based identification methods.

In the second group, spoligotyping is the gold-standard method for genotyping of MTC isolates, followed by MIRU-VNTR (Haddad, Masselot, \& Durand, 2004). Since the publications of spoligotyping in 1997 and MIRU-VNTR in 2006, both have been increasingly used, reaching a similar number of occurrences in the last years $(57.9 \%$ and $42.1 \%$, respectively), indicating an increased combination of both approaches, a trend that is observed across all continents (Fig. 6b).

More recently, since 2012, studies started to implement whole-genome sequencing (WGS) analyses $(n=20)$, mainly in Europe $(n=7)$ and North America $(n=6)$, to differentiate at a finescale MTC isolates and to try to infer epidemiological relationships (e.g. (Lasserre et al., 2018; Orloski et al., 2018; Price-Carter et al., 2018)).

Additionally, studies using network-based analyses have progressively been implemented in the animal TB field ( $n=49$ publications), in particular in Europe $(n=29)$, highlighting the scientific recognition of the importance of animal movement, animal interactions, and disease epidemiology, in several TB settings and scenarios.

Finally, works concerning epidemiological models ( $n=80$ publications) have progressively accumulated in the past two decades, being Europe $(n=58)$ the continent with most publications. The awareness of animal TB complexity, and the notion that different variables impact different disease settings, evidenced the necessity of developing transmission models, to inform control actions.

\subsection{Hosts}


Most studies reported animal TB in farmed animals (90.4\%), followed by infection in zoo animals $(6.7 \%)$ and pets $(2.9 \%)$. Most of the farmed animals retrieved in the publications belonged to the Bovidae family ( $82.1 \%)$, followed by Suidae (6.7\%), Cervidae (6.2\%), Camelidae (3.5\%) and other (1.5\%). The category denominated as "other" included a diverse range of host species, such as horse, broiler, lynx, donkey, and rabbit. Dogs, cats, rabbits, and ferrets were included in pets; while the zoo category encompassed over 20 different hosts, including elephants, giraffe, sea lion, or rhinoceros. Cattle was the most represented within the Bovidae family ( $82.3 \%$ ), followed by small ruminants $(12.3 \%)$, such as goat, sheep, and mouflon, and, finally, buffalo (3.6\%) and bison (1.8\%). The publication rate related to infection in farmed animals increased by 14 articles per year, while reports of infection in zoo and pet animals only started in 1994 and have had an increase of one article per year. Infection in Bovidae members has been reported with an increase of 11 articles per year, while the other families of farmed animals showed an increase of one article per year. Cattle-related articles showed an increase in publication rate of nine articles per year, followed by two articles per year in small ruminants, while works in the remaining groups of the Bovidae family show stagnation in publication. Europe was the continent where studies regarding zoo (number of studies $=23$ ) and pet $(n=9)$ animals were predominant.

Depending on the region of the world, cattle and buffalo are two important livestock species, bred and maintained for meat and milk purposes, and also as a draft power source. Cattle are widespread across the world, presenting highest densities in Asia (more specifically, in India, where such animals are not slaughtered), in Eastern African countries (particularly, Ethiopia), in Northern Europe and South America, while buffalos are concentrated in Asia, mostly in India and China (Gilbert et al., 2018; Robinson et al., 2014). The predominance of cattle as a host species in the set of retrieved publications, with works across all continents, was expected, not only by the prototypical association as an $M$. bovis reservoir but also because of its global distribution and economical relevance.

The small ruminant population includes goats and sheep that have distinctive global distributions, with the majority of works having origin in Europe $(n=39)$. The higher densities of goats were located in Asia (particularly, in India) and Africa, whereas the higher densities of sheep were found in Europe, central Africa, and Asia (Gilbert et al., 2018). The absence of studies concerning these hosts in South and North America might be related to less active production systems and, probably, with the allocation of research resources to other species with higher economic value. Only two studies in small ruminants originated from Asia, a vast geographic location with higher animal density than the rest of the world. So, it becomes evident that more research is a need in this geographical region and that research resources should be instigated in this field.

Studies related to Suidae members (including wild boar) were mainly performed in Europe $(n=25)$, followed by Africa $(n=6)$ and Oceania $(n=3)$. This asymmetrical distribution might be related with the global distribution of pig production systems, that is influenced by religious and cultural factors, with few occurring in predominantly Islamic countries and with higher density in East Asian (particularly, China), Eastern Pacific countries, and European countries (Gilbert et al., 2018; Robinson et al., 2014). Despite the high density of pig production systems in Asia, there is an absence of information concerning animal TB, so it could also be a relevant topic of research for this continent. Moreover, farmed wild boar, from the Suidae family, was also referred in European studies, but to a much lesser extent 
$(n=6)$. This species is often maintained in fenced areas (game farms) in several Western Mediterranean countries, such as Spain and France, where it is an important game species.

The Cervidae family has mainly been studied in Europe $(n=16)$ and North America $(n=14)$, which could also be associated with the occurrence of deer farms developed for meat production or hunting purposes.

Camelidae have only been studied in Europe $(n=11)$ and Africa $(n=8)$. Currently, these species are used for meat, milk, and hair fiber production and are distributed across South America, Northern Africa, East Europe, East Asia, and Australia, also being very popular as pet and zoo animals in Europe and North America (Zarrin et al., 2020), and for touristic purposes in the Canary Islands and Morocco. Climate change and the transformation of environments towards arid conditions might change the importance of this species in the production systems (Zarrin et al., 2020), therefore TB in camelids could be an important field of research in the future.

\subsection{Infectious agents}

498 The retrieved publications reported infection by seven MTC species or ecotypes: $M$. bovis (76.8\%), M. caprae (9.1\%), M. tuberculosis (8.9\%), M. pinnipedii (2.5\%), M. africanum (1.4\%), M. microti $(0.9 \%)$, and $M$. orygis $(0.5 \%)$. An increase in publication rate of 9 articles per year related with $M$. bovis studies and of 1 article per year for $M$. tuberculosis- and $M$. caprae-related works were observed. The increasing number of publications reporting infection with $M$. tuberculosis in livestock animals is probably related to an augmented interest at the livestock-human interface, namely reverse zoonosis (e.g. (Adesokan et al., 2019; Mittal et al., 2014; Prasad et al., 2005)). On the other hand, the increased number of publications reporting livestock infection with $M$. caprae is probably related with the recent recognition of M. caprae as a separate species from M. bovis (Aranaz et al., 2003), together with the increasing interest in livestock members other than cattle (e.g. goats are referred as M. caprae main hosts (Rodríguez et al., 2011)).

The above-mentioned MTC species were predominant in farmed animals, except for $M$. pinnipedii that was mostly reported in zoo animals $(n=10)$, while only $M$. bovis has been reported in pets. Reports on M. microti and M. africanum were restricted to farmed animals, with the former being mainly registered in Camelidae members $(n=3)$ and the latter exclusive of cattle $(n=6)$. All other MTC species were predominantly found in Bovidae members. Published works on Cervidae members were limited to M. bovis infection. Except for $M$. microti, the remaining MTC species were more prevalent in cattle. M. bovis was the most prevalent MTC species across all Bovidae members.

$M$. bovis was the most reported infectious agent across all continents, whereas $M$. orygis was exclusively reported in Asia and M. africanum in Africa. M. bovis, M. caprae, M. microti, and M. pinnipedii were predominant in Europe, while M. tuberculosis was predominant in Africa.

In the articles using spoligotyping as a genotyping technique, the top 5 most prevalent profiles within each MTC ecotype were registered, per article, and analyzed. M. bovis was predominantly identified as SB0121 (10.2\% of articles) and SB0120 (7.3\%), M. caprae as SB0157 (19.5\%) and SB0418 (19.5\%), and M. tuberculosis as SIT53 (20.0\%) and SIT149 (11.4\%). M. microti, M. orygis, and M. pinnipedii spoligotypes were only registered once: 
SB0118, SIT587, and SB1162, respectively. The extensive information regarding M. bovis also allowed performing geographic stratification by continent. SB0140 (18.2\%) was the most prevalent in Asia, SB0133 (6.7\%) and SB0944 (6.7\%) were equally recorded in Africa, SB0121 (15.9\%) and SB0120 (9.0\%) were the most frequently found in Europe, SB0673 (16.3\%) and SB0145 (14.3\%) were the most reported from North America, and SB0140 (14.3\%) and SB0121 (12.9\%) were the most stated in South America. Regarding Oceania, only four spoligotypes were registered, all with the same prevalence (25\%): SB0130, SB0140, SB1031, and SB1504. These results support the geographic stratification of molecular types across continents, possibly related to trade relationships and historical grounds, namely the colonial history of many former European colonial empires that fomented animal movement across colonies and territories.

\subsection{Disease transmission drivers}

The transmission drivers of animal TB most frequently mentioned by the authors of selected publications were analyzed. Management strategies (25.3\%) and livestock-wildlife interactions (22.3\%) were the most predominant studied drivers, followed by disease agents (19.2\%) and host features (18.5\%) (Fig.7). Management strategies include factors such as herd structure, herd type, or cohabitation of different species; the disease agent category considers differential prevalence of infection associated with different MTC members and with different MTC strains; and host features include sex, age, breed, or body condition as some of the considered aspects. Animal movements (5.8\%), livestock-human interactions (5.6\%), and environmental characteristics (3.3\%) also came to light (Fig. 7).

In Europe and Oceania, livestock-wildlife interactions (27.9\% and $42.1 \%$ ), management strategies $(27.4 \%$ and $26.3 \%$ ), and the disease agent (19.3\% and $26.3 \%)$ were the main drivers of transmission of animal TB. In Africa and North America, host features (35.5\% and $20.0 \%$ ) and management strategies $(22.7 \%$ and $25.7 \%)$ were reported as being the most predominant (Fig. 7). In Asia, both host features (42.1\%) and disease agent (26.3\%) were the most referred drivers, while in South America, disease agents (36.4\%), management strategies (27.3\%), and livestock-human interactions (22.7\%) were the major drivers (Fig. 7).

In agreement with these perceptions and results, control and eradication programs of animal TB in most countries have as main targets the improvement of management strategies, such as biosecurity measures and herd-related features, and restriction to livestock-wildlife interactions by fencing and application of selective feeders that allow the access to food by one species, but restrict the access to a different species, due to the feeder design (Pereira, Reis, Ramos, \& Cunha, 2020). However, the disease agent, such as different MTC species and the underlying genetic heterogeneity of ecotypes, as well as host features, such as sex, age, and breed, are more difficult parameters to control in the field, with the agent being almost impossible to control and the host being, nowadays, controlled through genetic selection, by the increased exploitation of animal breeds more resistant to animal TB (Pereira et al., 2020). However, this genetic selection is still rarely applied and the real effect of genetics in animal TB resistance is still poorly understood and profited.

\subsection{Overview}


As global human population is expected to increase from approximately 7.8 billion in 2020 (worldometers.info, 2020) to approximately 9.2 billion by 2050 (UNDP, 2008), demands for animal-based protein have driven the implementation of more industrialized methods of animal-food production, leading to an increase of intensive husbandry with subsequent effects on the transmission of infectious agents. Industrial systems for livestock production are now characterized by large numbers of animals being raised mainly in confinement, with the goal of rapid population turnover at a single infrastructure/site.

With the unceasing rise of the human population, livestock production industry will need to evolve to quickly respond to demand, with predictions estimating that production probably will need to increase by $70 \%$ until 2050 (Derner et al., 2017) and that the request for livestock products will be largely concentrated in Asia, Africa, and South America.

From 1968 until 2018, cattle production globally increased 29\% and sheep by 22\%, while the population of goat, pig, and buffalo duplicated. Estimates are that these numbers will keep on growing until 2050 (Derner et al., 2017; FAO, 2020). However, these trends are not constant throughout the globe: Africa and Asia registered an increase in cattle, sheep, and goat populations, whilst pig production rose in Africa and buffalo in Asia. In Europe, North America, and Oceania, the opposite situation occurred, with a reduction or stagnation of the total population of different animal species (Derner et al., 2017; FAO, 2020). Although it is still kept at high levels, meat production has reached a plateau in high-income countries since the 2000s but it has grown by 68\%, 64\%, and 40\% in Africa, Asia, and South America, respectively. Growth in low- and middle-income countries is largely attributed to the transition to high-protein diets and, to a lesser extent, the increase of the human population in those regions.

On average and on a global level, estimated livestock losses due to infectious disease represent more than $20 \%$ of total losses (Derner et al., 2017), and since, in the future, infectious diseases will remain dynamic, the implemented surveillance system will need to be flexible and easily adapt to the incoming challenges (Derner et al., 2017). Furthermore, stakeholders will have to become aware of the benefits from risk-based approaches to early detection surveillance and by implementing effective strategies to improve farmer awareness and engagement with veterinary health services.

The expected differential growth between developed and developing countries carry different challenges to livestock production systems. In developed countries, many production systems are increasing their efficiency and environmental sustainability, such as a reduction in greenhouse gas emissions, while in developing countries the rapidly increasing demand for livestock products is favoring the intensification of production systems. The exponential growth of livestock production systems focusing on particular species and the increasing importance of others, like camelids, give an important opportunity to improve knowledge of animal TB epidemiology in hosts other than cattle, where information is scarce from continents other than Europe. Since livestock production will need to rapidly increase in Africa, Asia, and South America to accompany the population growth and the underlying animal-protein demand, these geographic locations that currently mostly support works on cattle might need to extend their fields of research, perhaps promoting international collaborations with countries with more experience in other hosts and epidemiological scenarios. 


\subsection{Limitations}

614 Several limitations related to the strategy to acquire information on the published works are 615 acknowledged. Firstly, the use of Web of Science and Google Scholar to recover the 616 academic literature implied the exclusion of all grey literature, such as books and reports. 617 Secondly, the use of topic, in the Web of Science, and title, in Google Scholar, as a section 618 to search, limited the indexation of literature not including the search terms in those sections. 619 Thirdly, the restriction to English and Portuguese articles leads to a linguistic bias, excluding 620 valid literature produced in other languages. Additionally, it is important to highlight that the 621 number of publications or citations of an author or journal was used as an objective proxy 622 for the scientific production and visibility of a given scientific topic and do not imply any 623 value judgment for the quality or relevance of a particular study, author, institution or 624 publication. We thus highlight that there are many meaningful and relevant publications and 625 authors that could not be ranked according to the established criteria.

626 Also, given the wide broad scope of research works in animal TB, instead of focusing all the 627 themes that gravitate around this disease, we opted to focus on a subset of topics related with 628 the epidemiological component of TB. This strategy and the underlying criteria will of course 629 not highlight nor cite all golden papers and renowned scientists working on animal TB.

630 Having mentioned these constraints, we believe that our findings offer a valid representation 631 of research outputs within this research field at a global level.

\section{Conclusions}

633 This study provides an overview of animal TB epidemiology research in livestock on a 634 worldwide scale, reporting valuable information related to publication numbers, countries, 635 sources, mammalian hosts, infectious agents, and drivers of transmission. During the four decades under study, several advances in trends and knowledge occurred. The obtained data show a general growth of this field of research, mainly reflected in the increase of scientific production and international collaboration across European and American countries. The literature is strongly based on European countries, performing short period studies $(<10$ years) on cattle infected with M. bovis. Apart from this dominance of studies from Europe as a whole, countries on the top 10 are grounded on Europe, Africa, and America (both North and South), reflecting the strong international collaboration network among those continents. Additionally, an increasing tendency of authors to publish in more global journals was detected, reflecting an increased interest of the scientific community in general for animal TB epidemiology. Moreover, a progressive increase in both livestock-wildlife interface and zoonosis-related publications was noticed, with both Oceania and Europe being more aware of, or better equipped to lead with, the importance of wildlife reservoirs in animal TB epidemiology and the One Health perspective. Studies from Africa focus on zoonosis and zooanthroponosis (reverse zoonotic disease transmission) components due to the poor livestock farming conditions and non-pasteurized milk. Furthermore, tuberculin-based methods, histopathology and nucleic acid-based methods were the more prevalent diagnosis tools, while spoligotyping and MIRU-VNTR were the most prevalent genotyping tools. An increase in both WGS and network analysis has occurred in the last few years, being an expected trend for the next decade, which may justify a literature reappraisal in the coming years. Finally, management strategies and livestock-wildlife interactions were the most 
656

\section{Conflict of Interest}

664 The authors declare that the research was conducted in the absence of any commercial or 665 financial relationships that could be perceived as a potential conflict of interest.

\section{Author Contributions}

667 MVC conceived the study. ACR, BR, and ACP analysed the data under the regular 668 supervision of MVC and wrote the first draft of the manuscript. MVC critically revised and 669 redirected all drafts. All authors gave intellectual input to the conceptualization of the final 670 manuscript.

\section{Data Availability Statement}

672 Data sharing is not applicable to this article as no new data were created or analysed in this 673 study.

\section{$6749 \quad$ Funding}

This work was funded by Programa Operacional de Competitividade e Internacionalização (POCI) (FEDER component), Programa Operacional Regional de Lisboa, and Fundação para a Ciência e a Tecnologia (FCT), Portugal, in the scope of project 'Colossus: Control Of tubercuLOsiS at the wildlife/livestock interface uSing innovative natUre-based Solutions' (ref. POCI-01-0145- FEDER- 029783) and strategic funding to cE3c and BioISI Research Units (UID/ BIA/00329/2020 and UID/Multi/04046/2020). ACR and ACP were supported by FCT through doctoral grants (PD/BD/128031/2016 and SFRH/BD/136557/2018).

\section{References}

Adesokan, H. K., Akinseye, V. O., Streicher, E. M., Van Helden, P., Warren, R. M., \& Cadmus, S. I. (2019). Reverse zoonotic tuberculosis transmission from an emerging Uganda I strain between pastoralists and cattle in South-Eastern Nigeria. BMC Veterinary Research, 15(1), 437. doi: 10.1186/s12917-019-2185-1

Alexander, K. A., Laver, P. N., Michel, A. L., Williams, M., van Helden, P. D., Warren, R. M., \& Gey van Pittius, N. C. (2010). Novel Mycobacterium tuberculosis complex 
pathogen, M. mungi. Emerg Infect Dis, 16(8), 1296-1299. doi:

Allix, C., Walravens, K., Saegerman, C., Godfroid, J., Supply, P., \& Fauville-Dufaux, M. (2006). Evaluation of the Epidemiological Relevance of Variable-Number TandemRepeat Genotyping of $<\mathrm{em}>$ Mycobacterium bovis $</ \mathrm{em}>$ and Comparison of the Method with IS<em $>6110</$ em $>$ Restriction Fragment Length Polymorphism Analysis and Spoligotyping. 44(6), 1951-1962. doi: 10.1128/JCM.01775-05 \%J Journal of Clinical Microbiology

Aranaz, A., Cousins, D., Mateos, A., \& Dominguez, L. (2003). Elevation of Mycobacterium tuberculosis subsp. caprae Aranaz et al. 1999 to species rank as Mycobacterium caprae comb. nov., sp. nov. Int J Syst Evol Microbiol, 53(Pt 6), 1785-1789. doi: 10.1099/ijs.0.02532-0

Aranaz, A., Liébana, E., Mateos, A., Dominguez, L., Vidal, D., Domingo, M., . . Cousins, D. (1996). Spacer oligonucleotide typing of Mycobacterium bovis strains from cattle and other animals: a tool for studying epidemiology of tuberculosis. J Clin Microbiol, 34(11), 2734-2740.

Aria, M., \& Cuccurullo, C. (2017). bibliometrix: An R-tool for comprehensive science mapping analysis. Journal of Informetrics, 11(4), 959-975. doi: https://doi.org/10.1016/j.joi.2017.08.007

Ayele, W. Y., Neill, S. D., Zinsstag, J., Weiss, M. G., \& Pavlik, I. (2004). Bovine tuberculosis: an old disease but a new threat to Africa. Int J Tuberc Lung Dis, 8(8), 924-937.

Bourne, I. S. G. o. C. T. C. P. J. (2007). Bovine TB: The Scientific Evidence A Science Base for a Sustainable Policy to Control TB in Cattle An Epidemiological Investigation into Bovine Tuberculosis.

Brites, D., Loiseau, C., Menardo, F., Borrell, S., Boniotti, M. B., Warren, R., . . Gagneux, S. (2018). A New Phylogenetic Framework for the Animal-Adapted Mycobacterium tuberculosis Complex. 9(2820). doi: 10.3389/fmicb.2018.02820

Brosch, R., Gordon, S. V., Marmiesse, M., Brodin, P., Buchrieser, C., Eiglmeier, K., . . . Cole, S. T. (2002). A new evolutionary scenario for the <em $>$ Mycobacterium tuberculosis</em> complex. 99(6), 3684-3689. doi: 10.1073/pnas.052548299\%J Proceedings of the National Academy of Sciences

Carruth, L., Roess, A. A., Mekonnen, Y. T., Melaku, S. K., Nichter, M., \& Salman, M. (2016). Zoonotic tuberculosis in Africa: challenges and ways forward. The Lancet, 388(10059), 2460-2461. doi: 10.1016/S0140-6736(16)32186-9

Cooke, M. M., Jackson, R., Coleman, J. D., \& Alley, M. R. (1995). Naturally occurring tuberculosis caused by Mycobacterium bovis in brushtail possums (Trichosurus vulpecula): II. Pathology. $N$ Z Vet J, 43(7), 315-321. doi: 10.1080/00480169./1995.35912

Corner, L. A. (2006). The role of wild animal populations in the epidemiology of tuberculosis in domestic animals: how to assess the risk. Vet Microbiol, 112(2-4), 303-312. doi: 10.1016/j.vetmic.2005.11.015 
Coscolla, M., Lewin, A., Metzger, S., Maetz-Rennsing, K., Calvignac-Spencer, S., Nitsche, A., . . L Leendertz, F. H. (2013). Novel Mycobacterium tuberculosis complex isolate from a wild chimpanzee. Emerg Infect Dis, 19(6), 969-976. doi: 10.3201/eid1906.121012

Cosivi, O., Grange, J. M., Daborn, C. J., Raviglione, M. C., Fujikura, T., Cousins, D., . . . Meslin, F. X. (1998). Zoonotic tuberculosis due to Mycobacterium bovis in developing countries. Emerg Infect Dis, 4(1), 59-70. doi: 10.3201/eid0401.980108

Cousins, D. V., Bastida, R., Cataldi, A., Quse, V., Redrobe, S., Dow, S., . . Bernardelli, A. (2003). Tuberculosis in seals caused by a novel member of the Mycobacterium tuberculosis complex: Mycobacterium pinnipedii sp. nov. Int J Syst Evol Microbiol, 53(Pt 5), 1305-1314. doi: 10.1099/ijs.0.02401-0

Cross, M., Heeren, A., Cornicelli, L. J., \& Fulton, D. C. (2018). Bovine Tuberculosis Management in Northwest Minnesota and Implications of the Risk Information Seeking and Processing (RISP) Model for Wildlife Disease Management. 5(190). doi: $10.3389 /$ fvets.2018.00190

Cvetnic, Z., Katalinic-Jankovic, V., Sostaric, B., Spicic, S., Obrovac, M., Marjanovic, S., . . . Vickovic, I. (2007). Mycobacterium caprae in cattle and humans in Croatia. Int J Tuberc Lung Dis, 11(6), 652-658.

Derner, J. D., Hunt, L., Filho, K. E., Ritten, J., Capper, J., \& Han, G. (2017). Livestock Production Systems. In D. D. Briske (Ed.), Rangeland Systems: Processes, Management and Challenges (pp. 347-372). Cham: Springer International Publishing.

Duarte, E. L., Domingos, M., Amado, A., \& Botelho, A. (2008). Spoligotype diversity of Mycobacterium bovis and Mycobacterium caprae animal isolates. Veterinary microbiology, 130(3), 415-421. doi: https://doi.org/10.1016/j.vetmic.2008.02.012

EFSA, E. F. S. A., \& ECDC, E. C. f. D. P. C. (2019). The European Union One Health 2018 Zoonoses Report. 17(12), e05926. doi: 10.2903/j.efsa.2019.5926

EU, E. C. (2020). Outcome of the evaluation procedure of the eradication, control and surveillance programmes submitted by Member States for Union financial contribution for 2020: list of the programmes technically approved and amount allocated to each programme.

FAO. (2011). ONE HEALTH: Food and Agriculture Organization of the United Nations Strategic Action Plan.

FAO. (2020). FAOSTAT statistical database. Retrieved 04/2020

Frothingham, R., \& Meeker-O'Connell, W. A. (1998). Genetic diversity in the Mycobacterium tuberculosis complex based on variable numbers of tandem DNA repeats. Microbiology, 144 ( Pt 5), 1189-1196. doi: 10.1099/00221287-144-5-1189

Gagneux, S. (2018). Ecology and evolution of Mycobacterium tuberculosis. Nat Rev Microbiol, 16(4), 202-213. doi: 10.1038/nrmicro.2018.8 
Gallagher, J., Monies, R., Gavier-Widen, M., \& Rule, B. (1998). Role of infected, nondiseased badgers in the pathogenesis of tuberculosis in the badger. Vet Rec, 142(26), 710-714. doi: 10.1136/vr.142.26.710

Gibbs, E. P. J. (2014). The evolution of One Health: a decade of progress and challenges for the future. 174(4), 85-91. doi: 10.1136/vr.g143\%J Veterinary Record

Gilbert, M., Mitchell, A., Bourn, D., Mawdsley, J., Clifton-Hadley, R., \& Wint, W. (2005). Cattle movements and bovine tuberculosis in Great Britain. Nature, 435(7041), 491496. doi: $10.1038 /$ nature 03548

Gilbert, M., Nicolas, G., Cinardi, G., Van Boeckel, T. P., Vanwambeke, S. O., Wint, G. R. W., \& Robinson, T. P. (2018). Global distribution data for cattle, buffaloes, horses, sheep, goats, pigs, chickens and ducks in 2010. Scientific Data, 5(1), 180227. doi: 10.1038/sdata.2018.227

Haddad, N., Masselot, M., \& Durand, B. (2004). Molecular differentiation of Mycobacterium bovis isolates. Review of main techniques and applications. Res Vet Sci, 76(1), 1-18. doi: 10.1016/s0034-5288(03)00078-X

Haddad, N., Ostyn, A., Karoui, C., Masselot, M., Thorel, M. F., Hughes, S. L., . . Durand, B. (2001). Spoligotype diversity of Mycobacterium bovis strains isolated in France from 1979 to 2000. Journal of Clinical Microbiology, 39(10), 3623-3632. doi: 10.1128/JCM.39.10.3623-3632.2001

Humblet, M. F., Boschiroli, M. L., \& Saegerman, C. (2009). Classification of worldwide bovine tuberculosis risk factors in cattle: a stratified approach. Vet Res, 40(5), 50. doi: $10.1051 /$ vetres/2009033

Kamerbeek, J., Schouls, L., Kolk, A., van Agterveld, M., van Soolingen, D., Kuijper, S., . . . van Embden, J. (1997). Simultaneous detection and strain differentiation of Mycobacterium tuberculosis for diagnosis and epidemiology. Journal of Clinical Microbiology, 35(4), 907-914.

Kipar, A., Burthe, S. J., Hetzel, U., Rokia, M. A., Telfer, S., Lambin, X., . . Bennett, M. (2014). Mycobacterium microti tuberculosis in its maintenance host, the field vole (Microtus agrestis): characterization of the disease and possible routes of transmission. Vet Pathol, 51(5), 903-914. doi: 10.1177/0300985813513040

Kubica, T., Rüsch-Gerdes, S., \& Niemann, S. (2003). Mycobacterium bovis subsp. caprae caused one-third of human M. bovis-associated tuberculosis cases reported in Germany between 1999 and 2001. Journal of Clinical Microbiology, 41(7), 30703077. doi: 10.1128/jcm.41.7.3070-3077.2003

Lasserre, M., Fresia, P., Greif, G., Iraola, G., Castro-Ramos, M., Juambeltz, A., . . Berná, L. (2018). Whole genome sequencing of the monomorphic pathogen Mycobacterium bovis reveals local differentiation of cattle clinical isolates. Bmc Genomics, 19(1), 2. doi: 10.1186/s12864-017-4249-6

Miller, R. S., \& Sweeney, S. J. (2013). Mycobacterium bovis (bovine tuberculosis) infection in North American wildlife: current status and opportunities for mitigation of risks of further infection in wildlife populations. Epidemiology and Infection, 141(7), 1357-1370. doi: 10.1017/S0950268813000976 
813

814

815

816

817

818

819

820

821

822

823

824

825

826

827

828

829

830

831

832

833

834

835

836

837

838

839

840

841

842

843

844

845

846

847

848

849

850

851

Mittal, M., Chakravarti, S., Sharma, V., Sanjeeth, B. S., Churamani, C. P., \& Kanwar, N. S. (2014). Evidence of Presence of Mycobacterium tuberculosis in Bovine Tissue Samples by Multiplex PCR: Possible Relevance to Reverse Zoonosis. 61(2), 97104. doi: 10.1111/tbed.12203

Mostowy, S., Cousins, D., \& Behr, M. A. (2004). Genomic interrogation of the dassie bacillus reveals it as a unique RD1 mutant within the Mycobacterium tuberculosis complex. Journal of Bacteriology, 186(1), 104-109. doi: 10.1128/jb.186.1.104109.2003

Müller, B., Dürr, S., Alonso, S., Hattendorf, J., Laisse, C. J. M., Parsons, S. D. C., . . . Zinsstag, J. (2013). Zoonotic Mycobacterium bovis-induced tuberculosis in humans. Emerg Infect Dis, 19(6), 899-908. doi: 10.3201/eid1906.120543

Neto, J., Silveira, G., Rosa, B., Gonçalves, V., Gris-Filho, J. H. H., Amaku, M., . . Lage, A. (2016). Analysis of 15 years of the National Program for the Control and Eradication of Animal Brucellosis and Tuberculosis, Brazil. Semina: Ciências Agrárias, 37, 3385-3402. doi: 10.5433/1679-0359.2016v37n5Supl2p3385

OIE. (2018). Bovine tuberculosis Manual of Diagnostic Tests and Vaccines for Terrestrial Animals.

Orloski, K., Robbe-Austerman, S., Stuber, T., Hench, B., \& Schoenbaum, M. (2018). Whole Genome Sequencing of Mycobacterium bovis Isolated From Livestock in the United States, 1989-2018. 5(253). doi: 10.3389/fvets.2018.00253

Ortiz, A. P., Gutiérrez-Reyes, J. A., Velázquez, E. F., Reyes Escalona, G. A., \& Selva Hernández, E. T. (2014). Bovine tuberculosis eradication program in Mexico Zoonotic Tuberculosis (pp. 291-308).

Palmer, M. V., \& Waters, W. R. (2006). Advances in bovine tuberculosis diagnosis and pathogenesis: what policy makers need to know. Veterinary microbiology, 112(2-4), 181-190. doi: 10.1016/j.vetmic.2005.11.028

Pereira, A. C., Reis, A. C., Ramos, B., \& Cunha, M. V. (2020). Animal tuberculosis: Impact of disease heterogeneity in transmission, diagnosis and control. Transbound Emerg Dis., 00, 1- 19. doi: 10.1111/tbed.13539

Prasad, H. K., Singhal, A., Mishra, A., Shah, N. P., Katoch, V. M., Thakral, S. S., . . Acharya, S. K. (2005). Bovine tuberculosis in India: Potential basis for zoonosis. Tuberculosis, 85(5), 421-428. doi: https://doi.org/10.1016/j.tube.2005.08.005

Price-Carter, M., Brauning, R., de Lisle, G. W., Livingstone, P., Neill, M., Sinclair, J., . . . Collins, D. M. (2018). Whole Genome Sequencing for Determining the Source of Mycobacterium bovis Infections in Livestock Herds and Wildlife in New Zealand. 5(272). doi: 10.3389/fvets.2018.00272

Reis, A. C., Albuquerque, T., Botelho, A., \& Cunha, M. V. (2020). Polyclonal infection as a new scenario in Mycobacterium caprae epidemiology. Veterinary microbiology, 240, 108533. doi: 10.1016/j.vetmic.2019.108533 
852

853

854

855

856

857

858

859

860

861

862

863

864

865

866

867

868

869

870

871

872

873

874

875

876

877

878

879

880

881

882

883

884

885

886

887

888

889

890

891

Robinson, T. P., Wint, G. R. W., Conchedda, G., Van Boeckel, T. P., Ercoli, V., Palamara, E., ... Gilbert, M. (2014). Mapping the Global Distribution of Livestock. PLOS ONE, 9(5), e96084. doi: 10.1371/journal.pone.0096084

Rodriguez-Campos, S., Smith, N. H., Boniotti, M. B., \& Aranaz, A. (2014). Overview and phylogeny of Mycobacterium tuberculosis complex organisms: implications for diagnostics and legislation of bovine tuberculosis. Res Vet Sci, 97 Suppl, S5-s19. doi: 10.1016/j.rvsc.2014.02.009

Rodríguez, E., Sánchez, L. P., Pérez, S., Herrera, L., Jiménez, M. S., Samper, S., \& Iglesias, M. J. (2009). Human tuberculosis due to Mycobacterium bovis and M. caprae in Spain, 2004-2007. Int J Tuberc Lung Dis, 13(12), 1536-1541.

Rodríguez, S., Bezos, J., Romero, B., de Juan, L., Álvarez, J., Castellanos, E., . . . Monitoring of Animal, T. (2011). Mycobacterium caprae infection in livestock and wildlife, Spain. Emerg Infect Dis, 17(3), 532-535. doi: 10.3201/eid1703.100618

Schmitt, S. M., O'brien, D. J., Bruning-Fann, C. S., \& Fitzgerald, S. D. (2002). Bovine Tuberculosis in Michigan Wildlife and Livestock. 969(1), 262-268. doi: 10.1111/j.1749-6632.2002.tb04390.x

Sibhat, B., Asmare, K., Demissie, K., Ayelet, G., Mamo, G., \& Ameni, G. (2017). Bovine tuberculosis in Ethiopia: A systematic review and meta-analysis. Prev Vet Med, 147, 149-157. doi: 10.1016/j.prevetmed.2017.09.006

Smith, N. H. (2012). The global distribution and phylogeography of Mycobacterium bovis clonal complexes. Infect Genet Evol, 12(4), 857-865. doi: 10.1016/j.meegid.2011.09.007

Smith, N. H., Dale, J., Inwald, J., Palmer, S., Gordon, S. V., Hewinson, R. G., \& Smith, J. M. (2003). The population structure of Mycobacterium bovis in Great Britain: clonal expansion. Proc Natl Acad Sci U S A, 100(25), 15271-15275. doi: 10.1073/pnas.2036554100

Smith, N. H., Kremer, K., Inwald, J., Dale, J., Driscoll, J. R., Gordon, S. V., . . Smith, J. M. (2006). Ecotypes of the Mycobacterium tuberculosis complex. J Theor Biol, 239(2), 220-225. doi: 10.1016/j.jtbi.2005.08.036

Thoen, C., Lobue, P., \& de Kantor, I. (2006). The importance of Mycobacterium bovis as a zoonosis. Veterinary microbiology, 112(2-4), 339-345. doi: 10.1016/j.vetmic.2005.11.047

Thornton, P. K. (2010). Livestock production: recent trends, future prospects. Philosophical transactions of the Royal Society of London. Series B, Biological sciences, 365(1554), 2853-2867. doi: 10.1098/rstb.2010.0134

UNDP, U. N. D. P. (2008). Annual Report.

van Ingen, J., Rahim, Z., Mulder, A., Boeree, M. J., Simeone, R., Brosch, R., \& van Soolingen, D. (2012). Characterization of Mycobacterium orygis as M. tuberculosis complex subspecies. Emerg Infect Dis, 18(4), 653-655. doi: 10.3201/eid1804.110888 
892 van Soolingen, D., de Haas, P. E., Hermans, P. W., \& van Embden, J. D. (1994). DNA fingerprinting of Mycobacterium tuberculosis. Methods Enzymol, 235, 196-205.

895 Zarrin, M., Riveros, J. L., Ahmadpour, A., de Almeida, A. M., Konuspayeva, G., Vargas896 Bello-Pérez, E., . . . Hernández-Castellano, L. E. (2020). Camelids: new players in the international animal production context. Tropical Animal Health and Production. doi: 10.1007/s11250-019-02197-2 


\section{$900 \quad$ Figure captions}

901 Figure 1 - Retrieval and selection of articles for the review according to PRISMA guidelines.

902 Figure 2 - Trends in publication on tuberculosis in livestock.

903 [A] Annual median rise: (i) Global (1981-2020)= 10 articles per year; (ii) From 2003 to 2020

$904=24$ articles per year; [B] Number of publications according to the study period (black 1944-

905 2018) and the publication year (grey; 1981-2020).

906 Figure 3 - Worldwide Collaborations. Scientific production plotted in the spectral color

907 scale. Hot colors represent a higher number of publications. The higher number of 908 collaborative studies are represented by thicker lines.

909 Figure 4 - Trends in sources' field over time.

910 Figure 5 - Interface analyses. [A] Number of publications according to interface type over 911 time; [B] Percentage of publications according to interface type per continent.

912 Figure 6 - Trends in methodologies per continent. [A] Nucleic acid-based testing; 913 Biochemical testing; Histopathology; IFN-gamma testing; Tuberculin-based testing. [B] 914 Spoligotyping and MIRU-VNTR typing.

915 Figure 7 - Drivers of tuberculosis in livestock per continent.

916 

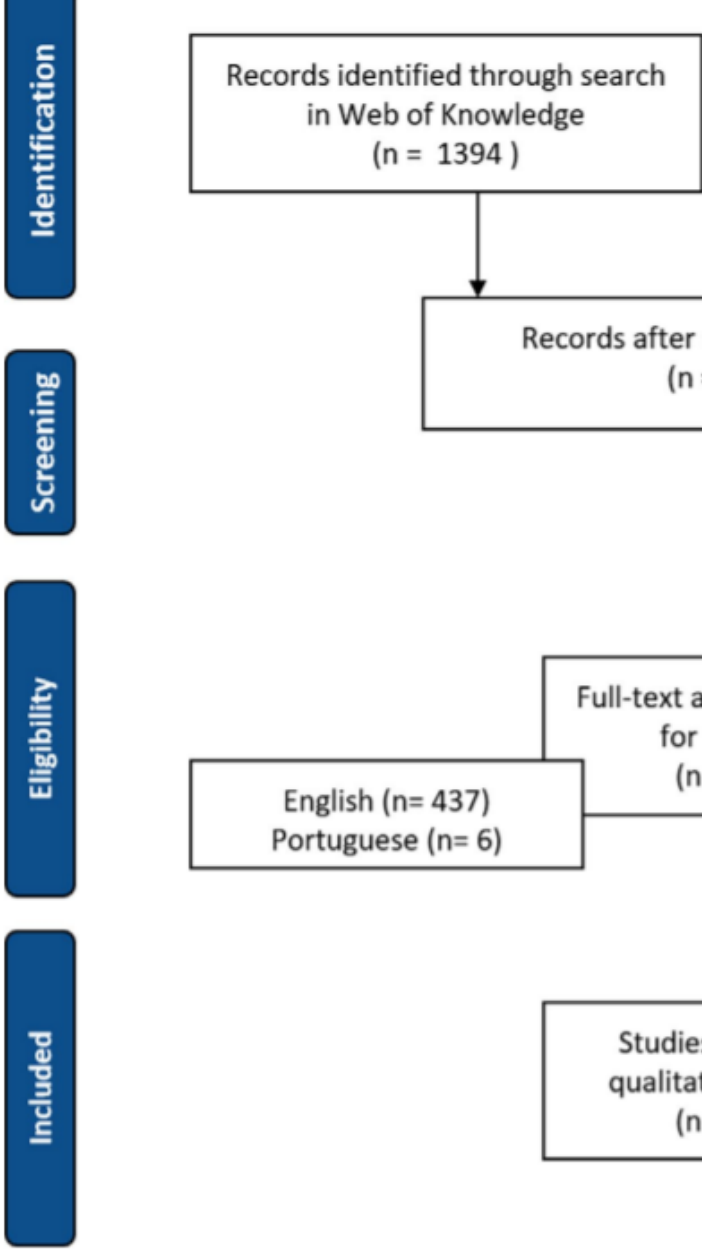

917

918 Figure 1
Excluded $(n=1000)$ : Inaccessible $(n=25)$; Review ( $n=63$ ); Out-ofscope $(n=884)$; Other language $(n=1)$;

Conference posters $(n=7)$;

Theses ( $n=19)$; Duplicate data $(n=1)$.

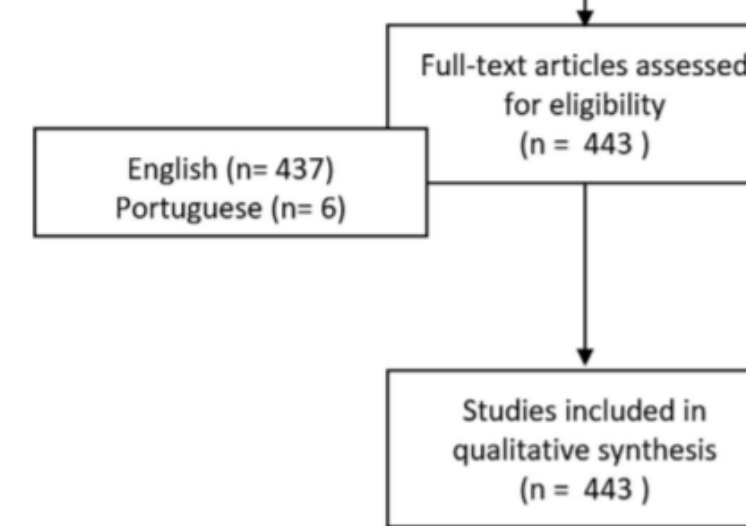

dditional records identified

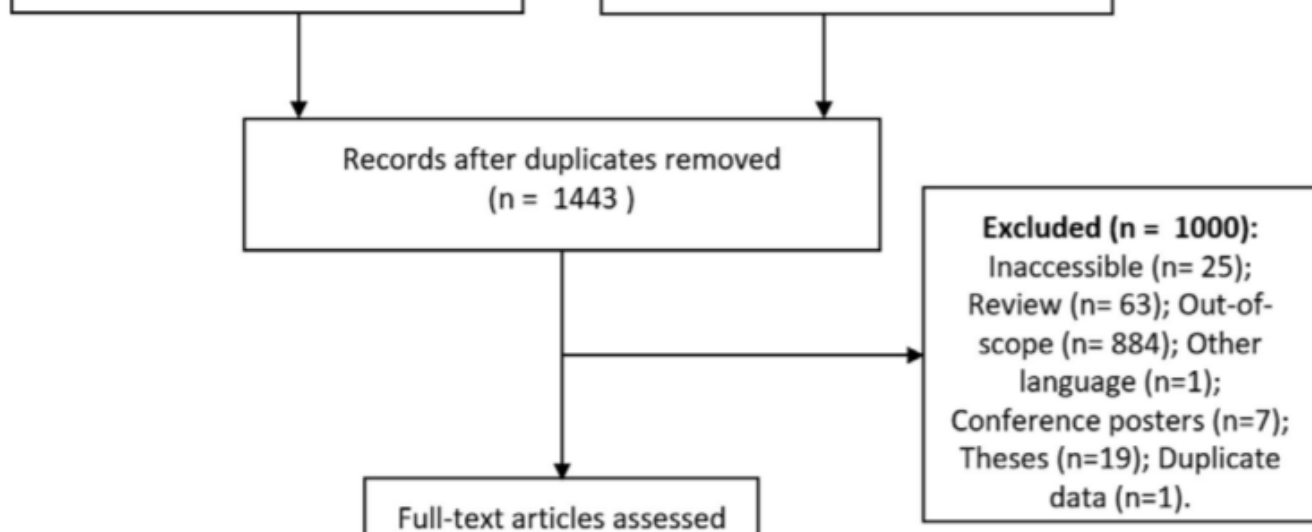

Studies included in

$$
\text { ( } n=443 \text { ) }
$$



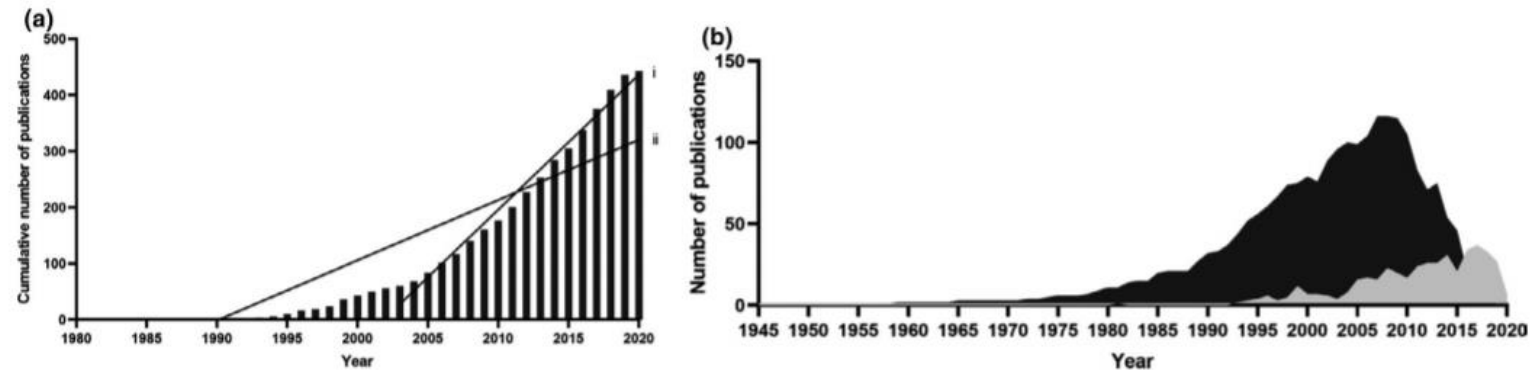

920 Figure 2 


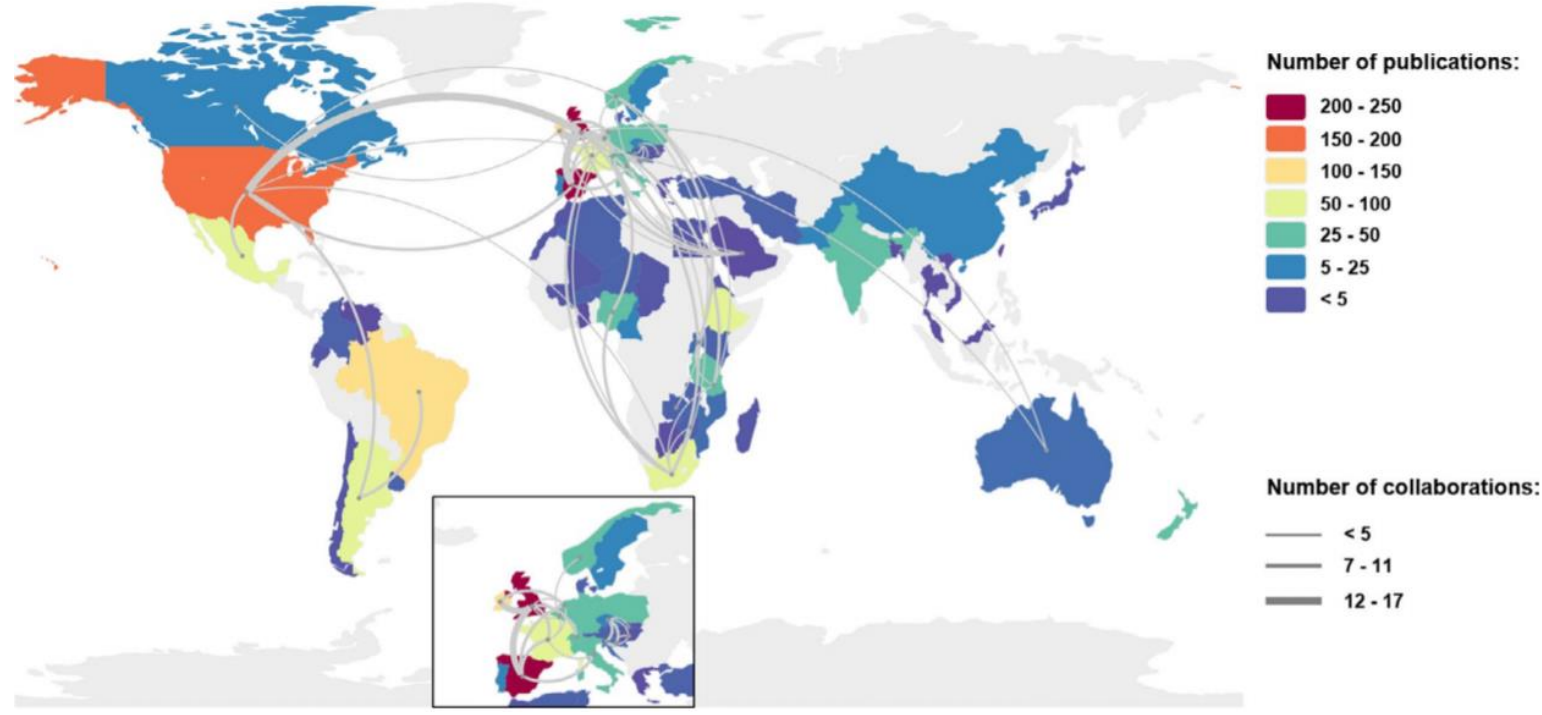

\section{Figure 3}




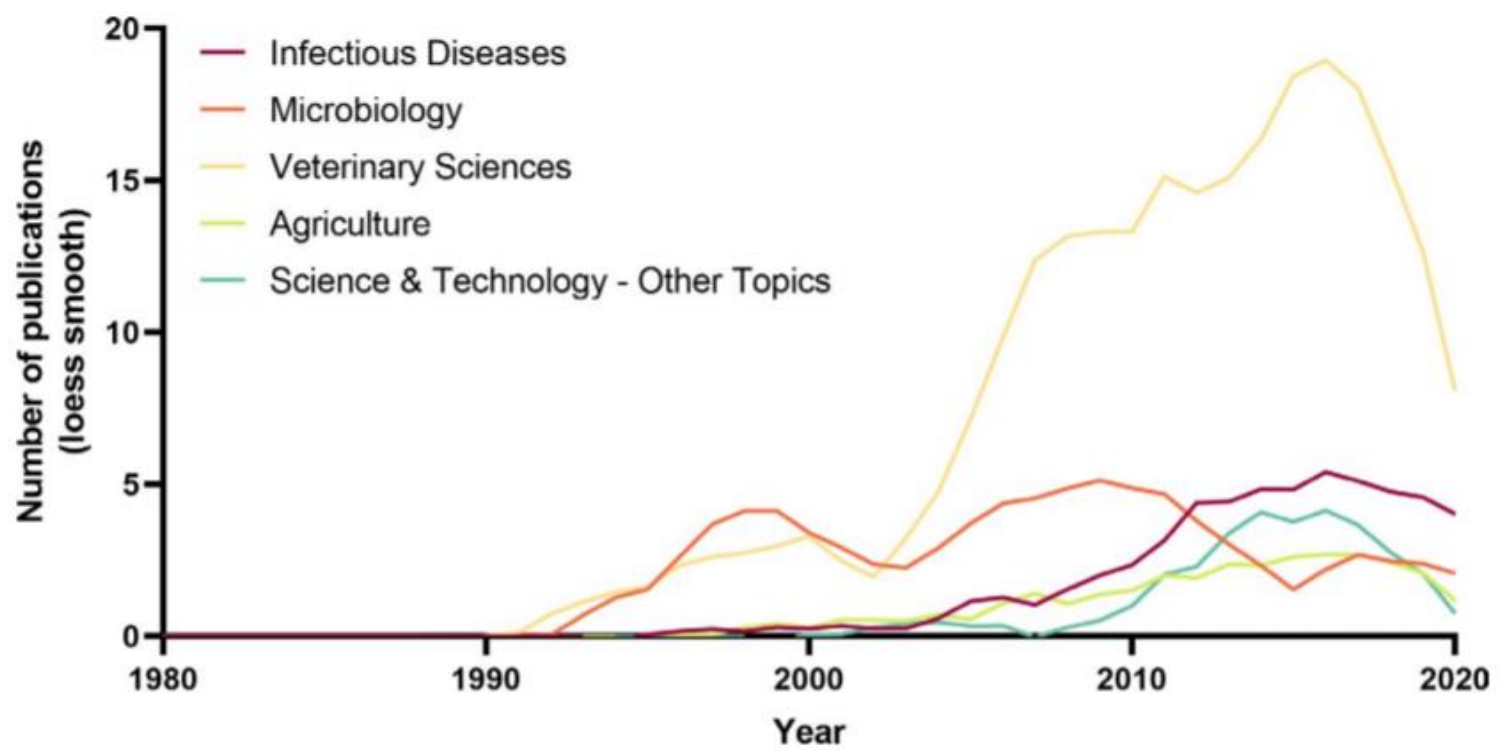

923

924 Figure 4 

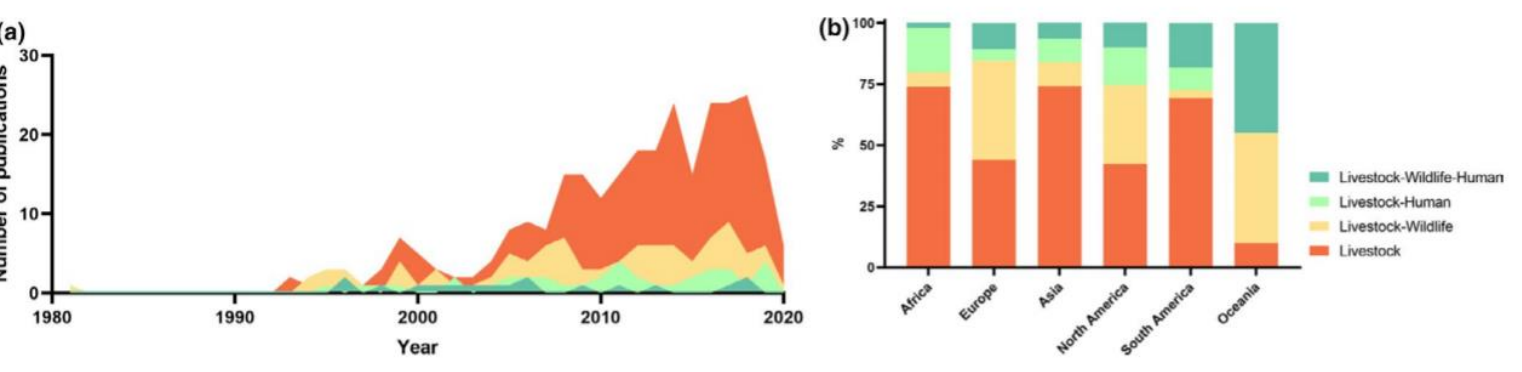

925

926

Figure 5 
(a)

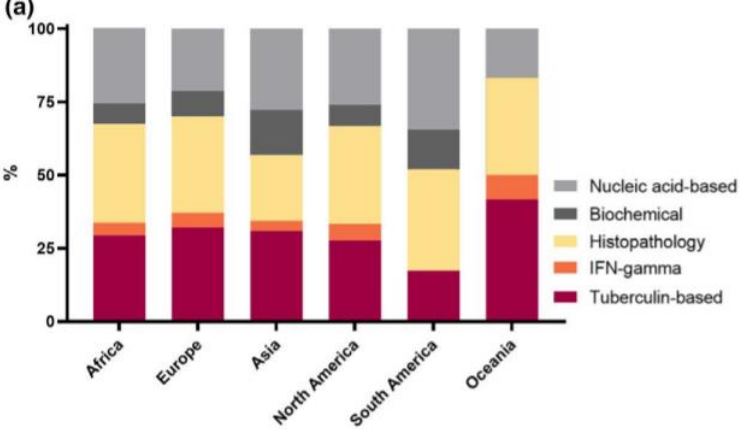

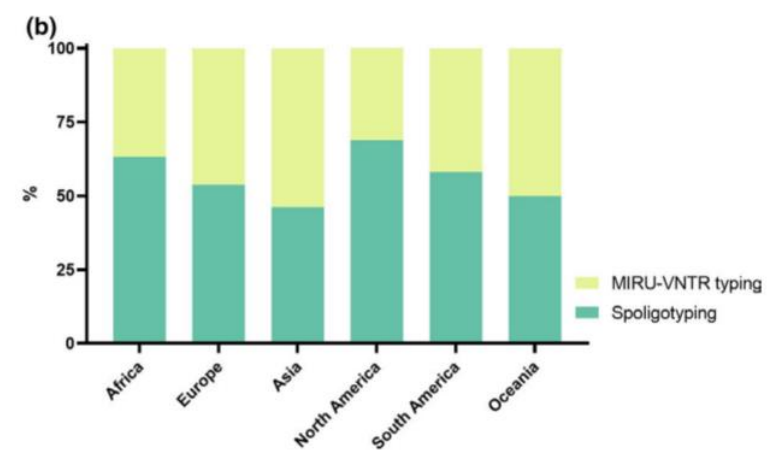

928 Figure 6 
929

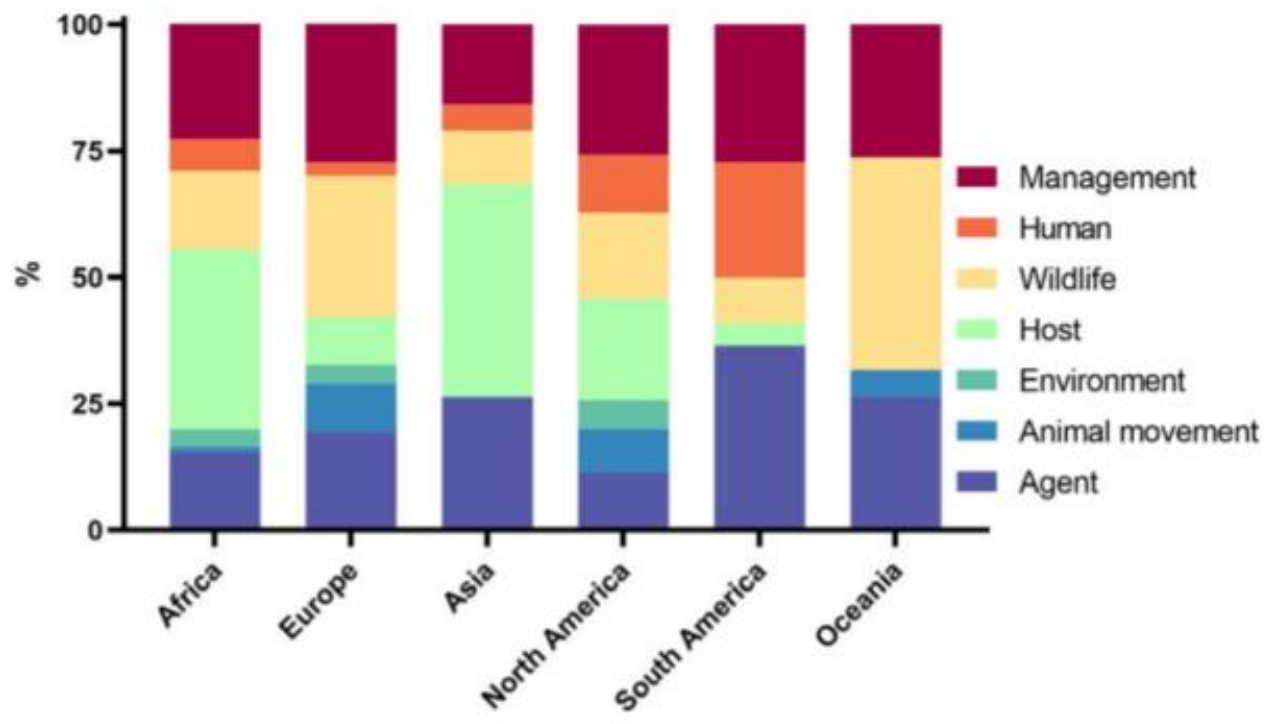

$930 \quad$ Figure 7 